\title{
Selected Results on the Transition from Short to Long Distance Physics at HERA
}

\author{
Jörg Gayler \\ DESY, Notkestrasse 85, 22603 Hamburg, Germany \\ E-mail: gayler@mail.desy.de
}

\begin{abstract}
Recent results from the HERA ep collider are discussed with emphasis on the transition from short to long distance phenomena in QCD. The results cover inclusive $e p$ scattering, inclusive diffractive scattering, vector meson production, and deeply virtual Compton scattering (DVCS).
\end{abstract}

\section{Introduction}

In electron proton $(e p)$ scattering at high energies, a wide spectrum of phenomena can be explored which are in principle covered by Quantum Chromodynamics (QCD), but which can not be described yet by rigorous detailed calculations. Whereas scattering processes involving hard interactions, such as production of jets, can be accessed by perturbative QCD (pQCD), large distance phenomena corresponding to soft interactions and involving only small scales, are mainly explored by experiment. Even the results on the proton structure function $F_{2}$, which are often considered as a triumph of QCD, provide both, a measurement of parton densities (pdf) at low scales $Q^{2}$ and indeed a successful pQCD test by the analysis of their evolution towards large $Q^{2}$. Here $Q^{2} \equiv-q^{2}$ is the 4-momentum squared of the exchanged virtual photon with $q=p_{e}-p_{e}^{\prime}$, where $p_{e}$ and $p_{e}^{\prime}$ are the 4-momenta of the incident and scattered electron respectively.

I present团 results on $F_{2}$ which are relevant in context of the discussions on saturation (see [1] and e.g. [2]), i. e. the damping of the rise of $F_{2}$ at high energies (small $x$ ) which is expected due to general arguments like unitarity, or more specifically in context of QCD due to increasing parton densities at high energies and gluon self interactions. Of particular interest are here the transition from deep inelastic scattering (DIS) to photo production $\left(Q^{2} \approx 0\right)$ and the high energy limit at fixed $Q^{2} \neq 0$.

Diffractive scattering, where the proton or photon (or dissociations of them) appear in the final state, is particularly interesting for the study of the transitions from short to long distance phenomena. At high momentum transfer or in case of jet production, such $e p$ interactions may be hard enough to justify pQCD calculations. On the other hand, the frequency of the reappearance of the initial state particles, in particular the $\ddagger$ talk presented at MAD’01, Antananarivo, October 2001 
proton, tells about the confining forces (confer the general discussion in [3]). Here I present results on inclusive diffractive ep scattering and also on specific final states where the virtual photon, emitted from the electron and interacting with the proton, is transformed into a particle with photon quantum numbers, like a real photon or a vector meson. Such quasi elastic reactions are in close relation to fully inclusive scattering, i.e. to the $F_{2}$ structure function, via the optical theorem.

Many other results on non-perturbative phenomena are available from HERA and could not be covered in this talk, like search for instantons, search for odderons [4], data on the photon structure, fragmentation, pion exchange and more.

\section{Inclusive reaction $(e p \rightarrow e X)$}

The proton structure function $F_{2}$ follows in a wide kinematic range the patterns expected from pQCD. In particular the $Q^{2}$ dependence can be described by DGLAP evolution of pdfs which are parametrised at low scales consistently with experimental data (e.g. [5, 6, 7]). However the measurements extend into the region of small $Q^{2}$ which correspond to transverse sizes of the interaction region well above $1 \mathrm{fm}$. Fig. 11 shows recent data 8, 9] on $F_{2}$ as function of $Q^{2}$ for different $W$, the invariant mass of the hadronic final state, with $W^{2}=(p+q)^{2}$, where $p$ is the 4-momentum of the incident proton. The region of low $Q^{2}$ is described by phenomenological models (here [9]). In

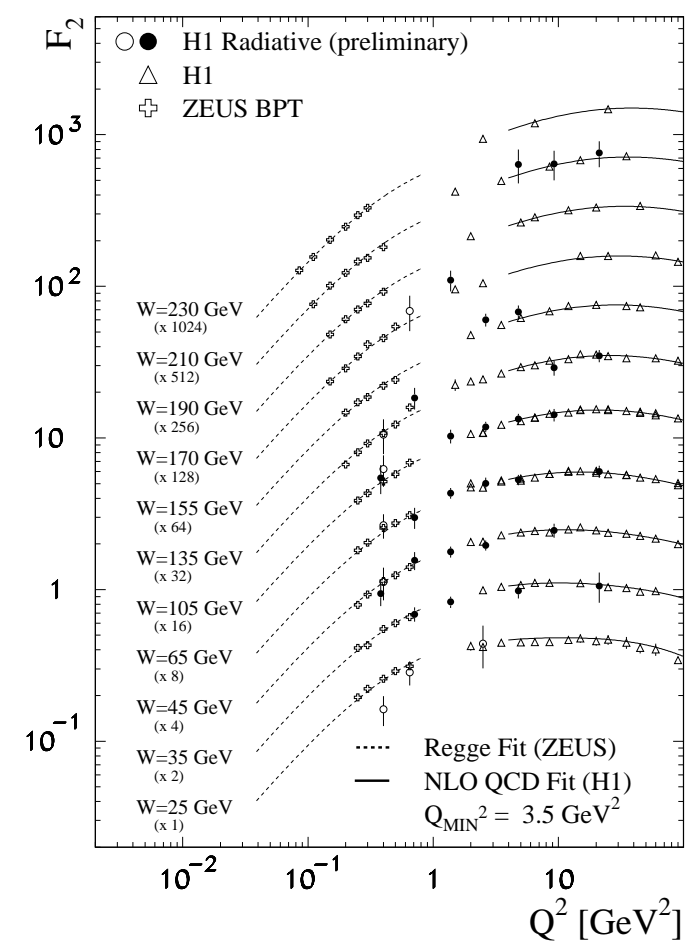

Figure 1. $F_{2}$ as function of $Q^{2}$ for different $W$.

the limiting case of photo production $\left(Q^{2}=0\right)$, the energy dependence is well described 
by Regge based models where the dependence on the total energy $W$ of the hadronic system is directly taken from soft hadronic reactions [10].

That $F_{2} \rightarrow 0$ for $Q^{2} \rightarrow 0$, as suggested by Fig. 1, can be expected from the relation

$$
F_{2}=1 / 4 \pi \alpha^{2} Q^{2} \sigma_{\text {tot }}^{\gamma^{*} p}
$$

with the total virtual photo production cross section $\sigma_{\text {tot }}^{\gamma^{*} p}$, which is constant for $Q^{2} \rightarrow 0$.

In contrast to the smooth energy dependence of $\sigma_{t o t}^{\gamma p}$, the energy dependence of $F_{2}$ is steep as demonstrated recently with high precision [5, 6] in terms of the $x$ dependence of $F_{2}$ [ 3 It is expected that this rise towards low $x$, which in pQCD analyses corresponds to an increase of the gluon density (see e.g. [5, 7]), will slow down at highest energies $($ small $x$ ) due to gluon-gluon interactions [1]. H1 studied the $x$ dependence in detail and evaluated [11] the derivative $\lambda=-\left(\partial \ln F_{2} / \partial \ln x\right)_{Q^{2}}$ using recent $F_{2}$ data [5]. Fig. 2] shows that $\lambda$ is constant within experimental uncertainties for fixed $Q^{2}$ in the range

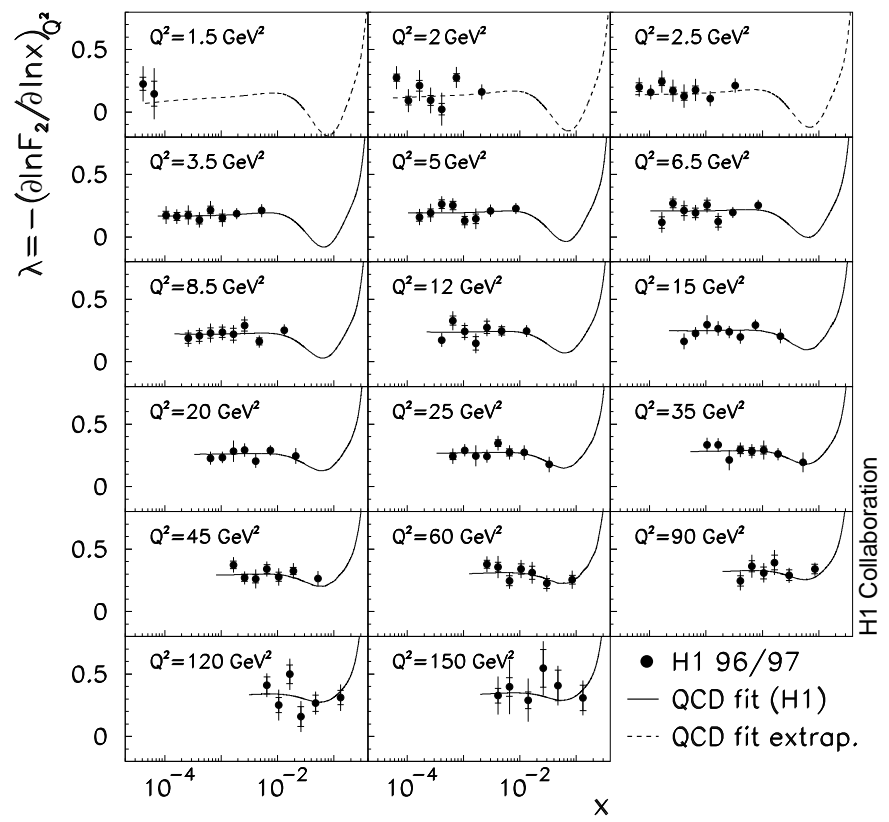

Figure 2. Derivative $\lambda=-\left(\partial \ln F_{2} / \partial \ln x\right)_{Q^{2}}$ for different $Q^{2}$ with $Q C D$ analysis of [5].

$x<0.01$, implying that the data are consistent with the power behaviour $F_{2}=\sim x^{-\lambda\left(Q^{2}\right)}$.

We conclude that no damping of the rise of $F_{2}$ is visible yet at present energies and $Q^{2} \gtrsim 2 \mathrm{GeV}^{2}$. In fact a very simple parametrisation

$$
F_{2}=c \cdot x^{-\lambda\left(Q^{2}\right)}, \text { with } \lambda\left(Q^{2}\right)=a \cdot \ln \left[Q^{2} / \Lambda^{2}\right]
$$

with $c \approx 0.18$ describes the low $x F_{2}$ data very well. A fit [11] to the H1 data [5] at $x<0.01, Q^{2}>3.5 \mathrm{GeV}^{2}$ yields $a=0.0481 \pm .0013 \pm .0037, \Lambda=292 \pm 20 \pm 51 \mathrm{MeV}$.

At very low $Q^{2}$ a flattening of the $Q^{2}$ dependence of $\lambda$ with $\lambda \approx 0.08$ at $Q^{2} \approx 0$ is expected from relation (1) considering the energy dependence of the total photo $\S$ The Bjorken variable $x=Q^{2} / 2 p q$ is related to $W$ by $W^{2}=Q^{2}(1 / x-1)$. 
production cross section. The latter can be written as $\sigma_{\text {tot }}^{\gamma p} \sim s^{\alpha_{\mathbb{P}}(0)-1}$ where $\alpha_{\mathbb{P}}(0)$, with $\alpha_{\mathbb{P}}(0)-1 \approx 0.08$, is the intercept of the pomeron trajectory invented to describe the high energy dependence of soft hadronic interactions [10]. Here $s$ is the total centre of mass energy squared with $s=W^{2} \sim Q^{2} / x$. The approach of $\lambda$ to the soft pomeron intercept at $Q^{2}<1 \mathrm{GeV}^{2}$ is indeed observed in an analysis by the ZEUS collaboration [12].

Also the derivative $\left(\partial F_{2} / \partial \ln Q^{2}\right)_{x}$ was previously discussed in context of the rise of $F_{2}$. If presented as function of $Q^{2}>3 \mathrm{GeV}^{2}$, a rise towards small $x$ is observed [5] consistent with pQCD DGLAP analyses. In contrast, if presented for fixed $W$, a decrease is observed [12] versus small $x$ which is however kinematically related to a decrease of $Q^{2}$.

In summary, we can state that the $F_{2}$ data for $Q^{2} \gtrsim 2 \mathrm{GeV}^{2}$ are well described by pQCD DGLAP analyses without any sign of damping of the rise versus low $x$. The decrease of $F_{2}$ versus small $Q^{2}$ (typically $Q^{2} \lesssim 1 \mathrm{GeV}^{2}$ ) corresponds to the smooth transition to photoproduction which is described by non-perturbative models used for soft hadronic interactions.

\section{Inclusive diffraction $(e p \rightarrow e Y X)$}

Interactions at HERA are called diffractive, when the exchanged virtual photon $\gamma^{*}$ and the incident proton dissociate into systems $X$ and $Y$ with colourless exchange indicated by $\mathbb{P}$ in Fig. 3 a. Such reactions are selected by demanding a large rapidity

a)

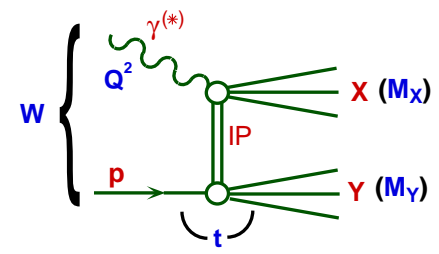

b)

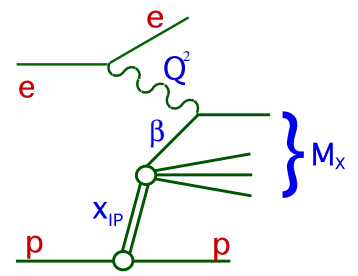

Figure 3. Inclusive diffractive reaction

gap which then separates the systems $X$ and $Y$, or by identifying the system $Y$ by direct measurement of a leading proton. In this talk new results from H1 [13] are presented which are described in terms of a QCD analysis of the diffractive exchange. The data of H1 [13 and ZEUS [14 are also compared to predictions of colour dipole models.

The interaction can be characterised kinematically by the variables

$$
x_{\mathbb{P}}=\frac{q \cdot\left(p-p^{\prime}\right)}{q \cdot p}=x_{(\mathbb{P} / p)} \quad, \quad \beta=\frac{Q^{2}}{q \cdot\left(p-p^{\prime}\right)}=x_{(\mathrm{q} / \mathbb{P})}
$$

where $p^{\prime}$ is the 4-momentum of the leading final state proton (system $Y$ ). $\beta$ can be interpreted as the momentum fraction of the exchanged object $\mathbb{P}$ which participates in the interaction with the virtual photon, $x_{\mathbb{P}}$ corresponds to the momentum fraction of the proton contributing to the colourless exchange (see Fig. $3 \mathrm{~b}$ ). The two variables are related by $x=x_{\mathbb{P}} \beta$. 
The data are presented in terms of the diffractive structure function

$$
F_{2}^{D(3)}\left(\beta, Q^{2}, x_{\mathbb{P}}\right)=\frac{\beta Q^{4}}{4 \pi \alpha^{2}\left(1-y+y^{2} / 2\right)} \frac{\mathrm{d} \sigma_{e p \rightarrow e X Y}}{\mathrm{~d} \beta \mathrm{d} Q^{2} \mathrm{~d} x_{\mathbb{P}}}
$$

defined in analogy to the fully inclusive structure function $F_{2}$ assuming $F_{L}^{D(3)}=0$. Here $y$ is the inelasticity given by $\left(p_{e}-p_{e}^{\prime}\right) \cdot p / p_{e} \cdot p$. The results are obtained by integration over $|t|=\left|\left(p-p^{\prime}\right)^{2}\right|<1 \mathrm{GeV}^{2}$.

It has been shown [15] that hard diffractive DIS can be factorised into probability distributions for partons $i$ for the case of an intact final state proton, which depend on $x_{\mathbb{P}}, t, x, Q^{2}$, and $\gamma^{*}$-parton cross sections $\sigma_{\gamma i}\left(x, Q^{2}\right)$. Soft hadron phenomenology, on the other hand, suggests "Regge factorisation". Here a universal pomeron flux $f_{\mathbb{P} / p}\left(x_{\mathbb{P}}, t\right)$ is assumed. The cross section can then be written as

$$
\sigma\left(\gamma^{*} p \rightarrow X p\right) \sim f_{\mathbb{P} / p}\left(x_{\mathbb{P}}, t\right) \otimes \sum_{i} f_{i / \mathbb{P}}\left(\beta, Q^{2}\right) \otimes \hat{\sigma}_{\gamma^{*} i}\left(\beta, Q^{2}\right)
$$

where the $f_{i}$, in the spirit of Ingelman and Schlein [16], are the densities of the partons which interact with the virtual photon.

The precise $\mathrm{H} 1$ data (Fig. (1) [13, which cover the range $6.5 \leq Q^{2} \leq 120 \mathrm{GeV}^{2}$ and $0.04 \leq \beta \leq 0.9$, are well described by a fit assuming Regge factorisation, with the result $\alpha_{\mathbb{P}}(0)=1.173 \pm 0.018$ (stat.) \pm 0.017 (syst. $)_{-0.035}^{+0.063}$ (model). Compatible results are obtained if the fit is performed in two separate $Q^{2}$ ranges.

We can state (see also [17):

- at $Q^{2} \gtrsim 1 \mathrm{GeV}^{2}, \alpha_{\mathbb{P}}(0)$ is above the soft pomeron expectation [10] of 1.08 ,

- $\alpha_{\mathbb{P}}(0)$ seems to grow slower in diffraction than in inclusive DIS (see eq.2).

In spite of the latter point, the energy dependencies are similar for diffractive and inclusive DIS. This corresponds to the different expected dependencies $\sim x^{2-2 \alpha_{\mathbb{P}}(t)}$ and $\sim x^{1-\alpha_{\mathbb{P}}(0)}=x^{-\lambda}$ respectively (see sections 1 and 2 ).

The results of a pQCD fit in terms of pomeron pdfs to the $\beta, Q^{2}$ dependence of the data are shown in Fig. $⿴$ for the case $x_{\mathbb{P}}=0.003$. The $\beta$ dependence is rather flat, the scaling violations in $\ln Q^{2}$ are positive up to large $\beta$, with the consequence that a large gluon component is required for the pdfs of the pomeron up to large $\beta$.

Quite differently from the approach of resolved pomeron models discussed above, colour dipole models (e.g. [18, 19, 20]) aim to describe the long distance features of the interaction by modeling an effective cross section for the interaction of $q \bar{q}$ pairs or $q \bar{q} g$ systems with the proton. Seen in the proton rest frame, these colour dipoles are produced perturbatively at large longitudinal distances $\sim 1 / m_{p} x$, well outside the proton for small $x$. The effective transverse size of these fluctuations is $<r^{2}>\sim 4 / Q^{2}$. In the model of Golec-Biernat and Wüsthoff [19] saturation of the dipole cross section at large radii, i.e. small $Q^{2}, p_{t}^{2}$, is introduced which at small $x$ is reached already at smaller radii.

Two colour dipole models [18, 19] are compared with the measured $\beta$ distributions of $x_{\mathbb{P}} F_{2}^{D(3)}$ for different $Q^{2}$ in Fig. 5. The general features of the data are described by 

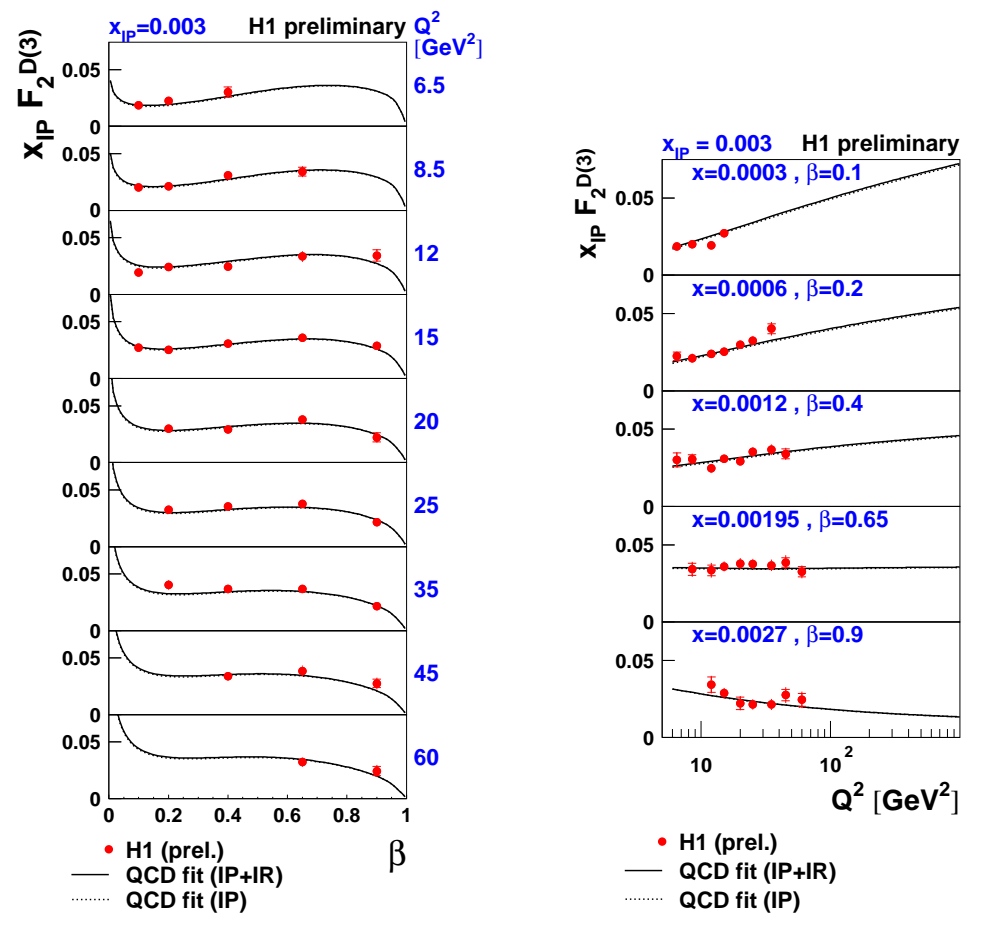

Figure 4. $x_{\mathbb{P}} F_{2}^{D(3)}$ as function of $\beta, Q^{2}$ and resolved $\mathbb{P}$ fit (6.8\% normalisation error of data not shown).
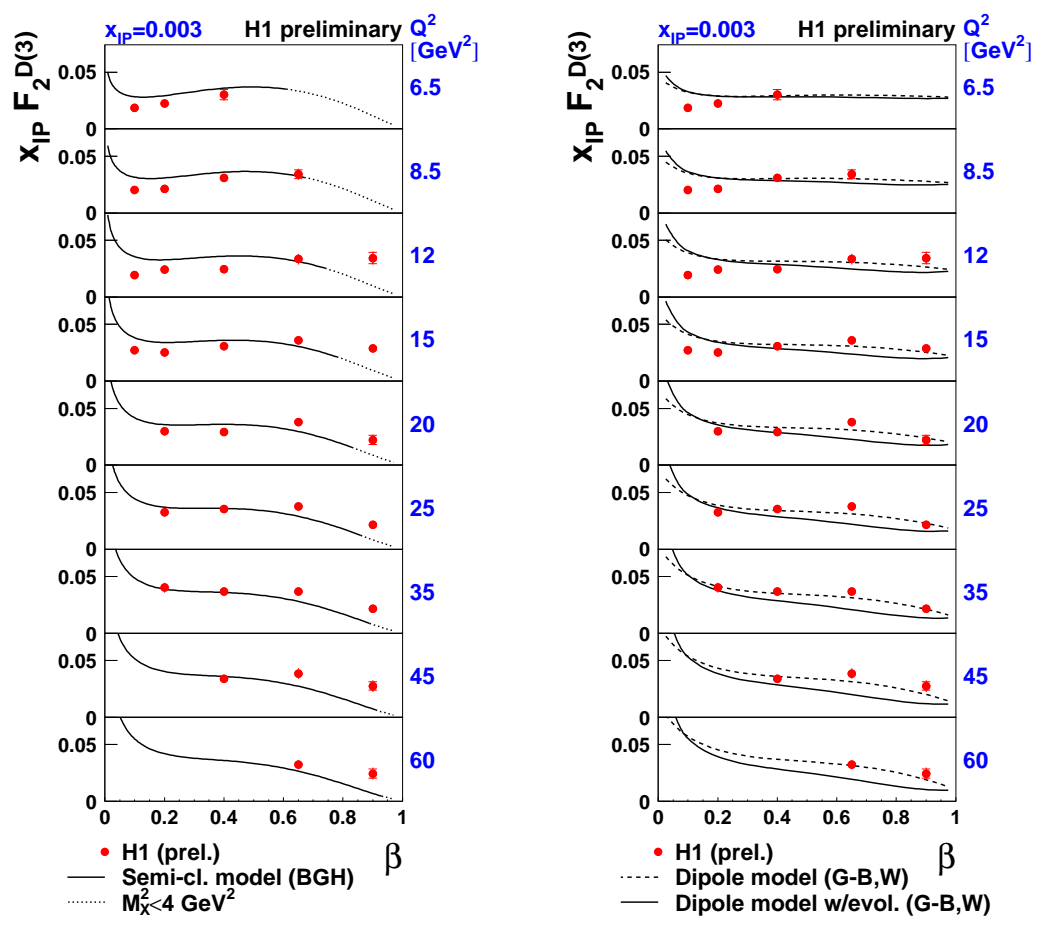

Figure 5. $x_{\mathbb{P}} F_{2}^{D(3)}$ as function of $\beta, Q^{2}$ and colour dipole models $[18,19,20]$. 
the models, but both are above the data at low $\beta$, low $Q^{2}$. The saturation model [19] contains a higher twist contribution at large $\beta$, but no improvement is achieved by a more advanced version involving $Q^{2}$ evolution [20].

The colour dipole models successfully predict the similarity of the energy dependencies of diffractive and fully inclusive ep DIS as shown in Fig. 6f where the ratio of the corresponding cross sections [14] as function of $W$ is compared with the saturation model [19]. The $q \bar{q} g$ component in the model is dominant at large $M_{X}$.

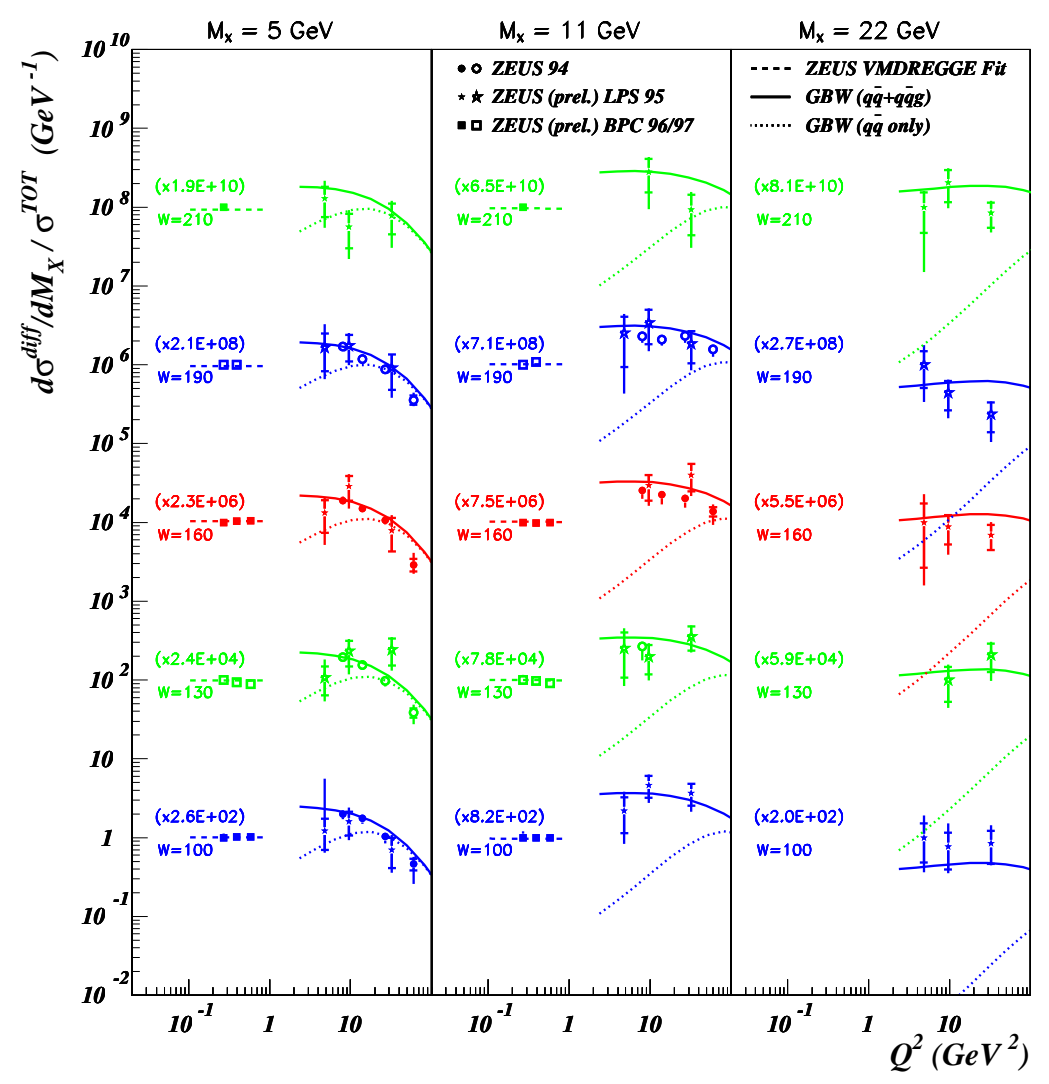

Figure 6. Ratio of diffractive and inclusive cross sections in $3 M_{X}$ ranges as function of $Q^{2}$ compared with the colour dipole model [19].

The main results on inclusive diffraction can be summarised as follows:

- The data are consistent with Regge factorisation.

- The effective $\alpha_{\mathbb{I P}}(0)$ is at large $Q^{2}$ larger than expected from soft hadronic interactions.

- The scaling violations $d F_{2}^{D 3} / d Q^{2}$ are positive up to large $\beta(>0.4)$ which is interpreted in DGLAP QCD analyses as a large gluon content in pomeron pdfs.

- Colour dipole models give a reasonable description of the main features of the data. 


\section{Exclusive Diffractive Reactions $(e p \rightarrow e V p, e p \rightarrow e \gamma p)$}

Exclusive vector meson and $\gamma$ production is particularly useful to gain understanding of diffractive processes. In these reactions the transverse interaction radius can be varied systematically by choosing different $Q^{2}$ and particle mass $M_{V}$.

The energy $(W)$ dependence gets steeper with both, increasing particle mass in photo production (see e.g. [21]) and also with increasing $Q^{2}$ as demonstrated for $\rho$ production in Fig. [7a which shows the exponent $\delta$ for fits to ZEUS data [22] according $\sigma \sim W^{\delta}$. In both cases the rise is steeper when the reactions get less peripheral. In fact an approximate scaling behaviour of vector meson production with the effective mass squared $Q^{2}+M_{V}^{2}$ can be observed (Fig. (7b). It should be noted that the figure shows the sum of longitudinal $\left(\sigma_{L}\right)$ and transverse $\left(\sigma_{T}\right)$ cross sections which are known to behave
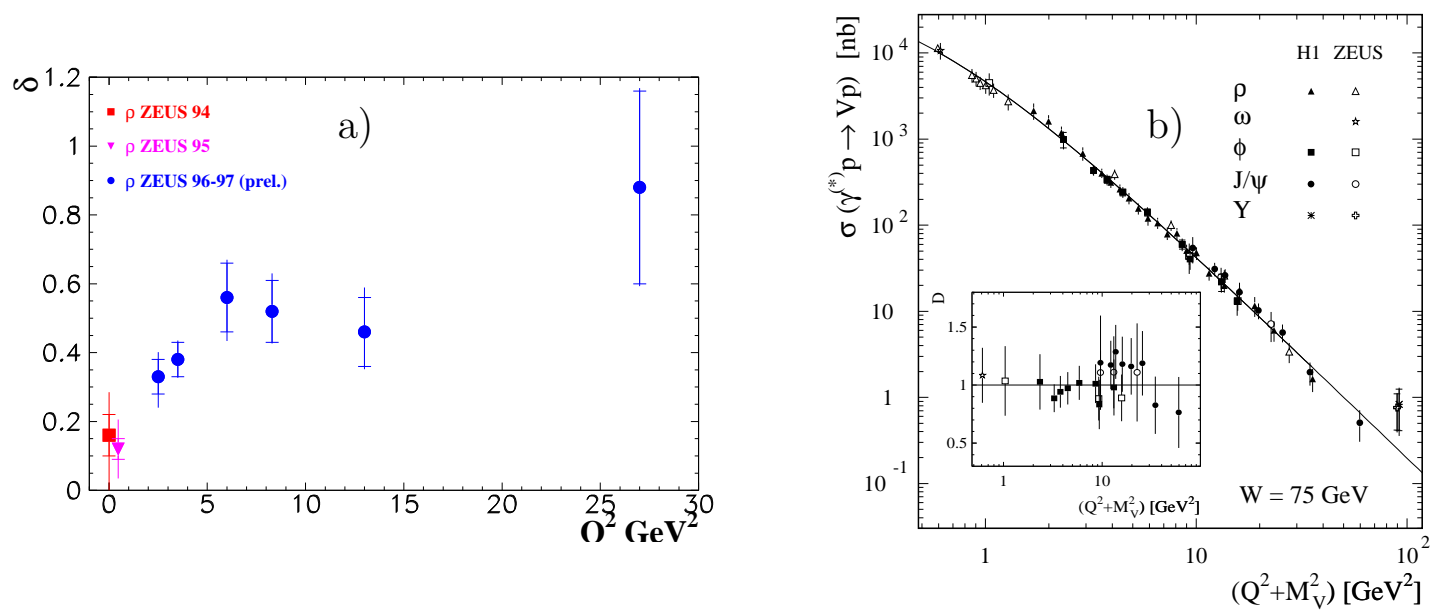

Figure 7. a) Exponent $\delta$ of from a fit $\sim W^{\delta}$ to $\rho$ production vs. $Q^{2}$. b) Vector meson production as function of $Q^{2}+M_{V}^{2}$ scaled by $\operatorname{SU}(4)$ factors $\rho^{0}: \omega: \Phi: J / \Psi=$ $9: 1: 2: 8$.

differently, and further, $J / \psi$ production seems to be somewhat above the expectation from the scaling behaviour, as seen in a more recent analysis [23].

The $t$ dependence $\sim \exp (-b|t|)$ of diffractive vector meson production yields a direct measure of the transverse size $r$ of the interaction region $\left(b \sim<r^{2}>\right)$. The HERA data (e.g. [24]) show indeed that $b$ decreases for increasing $Q^{2}+M_{V}^{2}$. It is however an interesting question, whether in vector meson production at large $Q^{2}$ or $M_{V}^{2}$, the effective size of the interaction region increases with energy as it is observed in soft hadronic interactions ("shrinkage" of the $t$-slope $b$ ). The available data on the energy dependence of $\rho$ and $J / \psi$ production at different $t$ indicate a slight increase of the interaction region with increasing energy [25], but less than expected from soft hadronic interactions.

First data on deep inelastic Compton scattering $\gamma^{*} p \rightarrow \gamma p$ (DVCS) have been presented recently [26, 27, 28, 29]. DVCS is a particularly clean process of lightquark scattering. The proton vertex is similar as in vector meson production, given 
in the simplest case by two exchanged gluons or quarks, but free of the complication by quark wave functions of the vector meson states. The simplicity of the process may give better access to the "skewed" or generalised parton density distributions in the proton than vector meson production. H1 [27] and ZEUS 29] subtracted the contribution of the purely electromagnetic Bethe Heitler $(\mathrm{BH})$ reaction, exploiting its different angular distribution. The resulting $Q^{2}$ and $W$ distributions are reasonably described by a leading order QCD model including non-perturbative elements [30] and a model based on pomeron and Reggeon exchange [31] (Fig.8). The HERMES and
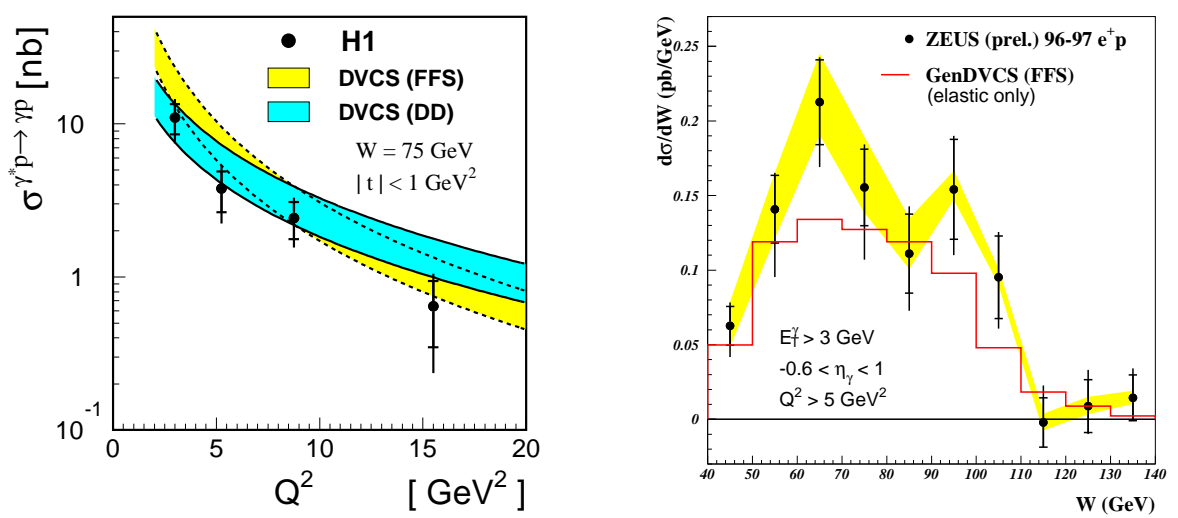

Figure 8. DVCS results as function of $Q^{2}$ and $W$ compared with a leading order QCD model (FFS [30]) and $\mathbb{P}$ and Reggion exchange (DD [31]).

CLAS collaborations determined the interference with the $\mathrm{BH}$ process by measuring the beam spin asymmetry. This field is rapidly developing: Next to leading order QCD calculations are meanwhile available [32] and more data from HERA are to be expected, in particular on the DVCS-BH interference.

\section{Conclusions}

- HERA provides information on the transition from short to long distance phenomena.

- The rise of $F_{2}$ versus small $x$ at $Q^{2} \gtrsim 2 \mathrm{GeV}^{2}$ shows no effect of saturation at present energies.

- The colour singlet exchange in diffractive reactions can be described by $\mathbb{P}$ parton densities ("resolved $\mathbb{P}$ "). The data require a large gluon component.

- The energy dependence of vector meson production is steep when objects of small transverse size $\left(J / \Psi, \rho^{0}\right.$ at high $\left.Q^{2}\right)$ are involved, but comparable to that of soft hadronic interactions in other cases.

- First results on deeply virtual Compton scattering (DVCS) are consistent with calculations based on leading order QCD and non-perturbative model assumptions. Future data will supply more information on generalised parton distributions. 


\section{Acknowledgments}

I am grateful to P. Schleper and M. Derrick for comments on the manuscript.

\section{References}

[1] L. V. Gribov, E. M. Levin and M. G. Ryskin, Nucl. Phys. B 188 (1981) 555; ibid Phys. Rept. 100 (1983) 1.

[2] H. Navelet, R. Peschanski and S. Wallon, Mod. Phys. Lett. A 9 (1994) 3393; E. Gotsman, E. Ferreira, E. Levin, U. Maor and E. Naftali, Phys. Lett. B 500 (2001) 87.

[3] J. Bartels and H. Kowalski, Eur. Phys. J. C 19 (2001) 693.

[4] J. Stiewe, these proceedings.

[5] C. Adloff et al. [H1 Collaboration], of Eur. Phys. J. C 21 (2001) 33.

[6] S. Chekanov et al. [ZEUS Collaboration], Eur. Phys. J. C 21 (2001) 443.

[7] A. D. Martin, R. G. Roberts, W. J. Stirling and R. S. Thorne, H. L. Lai et al. [CTEQ Collaboration], Eur. Phys. J. C 12 (2000) 375.

[8] H1 Collab., contributed paper EPS2001 (Budapest), Abstract 801 and references therein.

[9] J. Breitweg et al. [ZEUS Collaboration], Phys. Lett. B 487 (2000) 53.

[10] A. Donnachie and P. V. Landshoff, Phys. Lett. B 296 (1992) 227.

[11] C. Adloff et al. [H1 Collaboration], Phys. Lett. B 520 (2001) 183.

[12] ZEUS Collab., contributed paper EPS2001 (Budapest), Abstract 629.

[13] H1 Collab., contributed paper EPS2001 (Budapest), Abstract 808.

[14] A. Pellegrino, ZEUS Collab., DIS2001, Bologna.

[15] L. Trentadue and G. Veneziano, Phys. Lett. B 323 (1994) 201; A. Berera and D. E. Soper, Phys. Rev. D 50 (1994) 4328; M. Grazzini, L. Trentadue and G. Veneziano, Nucl. Phys. B 519 (1998) 394; J. C. Collins, Phys. Rev. D 57 (1998) 3051 [Erratum-ibid. D 61 (1998) 019902]

[16] G. Ingelman and P. E. Schlein, Phys. Lett. B 152 (1985) 256.

[17] ZEUS Collab., contributed paper ICHEP 2000 (Osaka), Abstract 875.

[18] W. Buchmuller, T. Gehrmann and A. Hebecker, Nucl. Phys. B 537 (1999) 477.

[19] K. Golec-Biernat and M. Wusthoff, Phys. Rev. D 59 (1999) 014017; ibid Phys. Rev. D 60 (1999) 114023.

[20] K. Golec-Biernat and M. Wusthoff, Eur. Phys. J. C 20 (2001) 313.

[21] L. Adamczyk for H1 and ZEUS, Nucl. Phys. Proc. Suppl. 99A (2001) 97.

[22] ZEUS Collab., EPS2001 (Budapest), Abstract 594, A. Kreisel, DIS2001, Bologna.

[23] ZEUS Collab., EPS2001 (Budapest), add. to abstracts 548/559/594.

[24] B. Mellado for H1 and ZEUS, ICHEP2000 (Osaka).

[25] C. Adloff et al. [H1 Collaboration], Phys. Lett. B 483 (2000) 23; ZEUS Collab., EPS2001 (Budapest), Abstracts 548,594.

[26] A. Airapetian et al. [HERMES Collaboration], Phys. Rev. Lett. 87 (2001) 182001.

[27] C. Adloff et al. [H1 Collaboration], Phys. Lett. B 517 (2001) 47.

[28] S. Stepanyan et al. [CLAS Collaboration], Phys. Rev. Lett. 87 (2001) 182002.

[29] ZEUS Collab., contributed paper EPS2001 (Budapest), Abstract 564.

[30] L. L. Frankfurt, A. Freund and M. Strikman, Phys. Rev. D 58 (1998) 114001 [Erratum-ibid. D 59 (1998) 119901].

[31] A. Donnachie and H. G. Dosch, Phys. Lett. B 502 (2001) 74.

[32] A. Freund and M. McDermott, arXiv:hep-ph/0111472. 